Kutzelmann, Sabine; Massler, Ute; Hendel, Robert

Das Projekt Mehrsprachiges Lesetheater: Ausgangslage, Zielsetzung und praxisrelevante Ergebnisse der Entwicklung

Mehrsprachiges Lesetheater. Handbuch zu Theorie und Praxis. Opladen; Berlin; Toronto : Verlag Barbara Budrich 2017, S. 7-14

Quellenangabe/ Reference:

Kutzelmann, Sabine; Massler, Ute; Hendel, Robert: Das Projekt Mehrsprachiges Lesetheater:

Ausgangslage, Zielsetzung und praxisrelevante Ergebnisse der Entwicklung - In: Mehrsprachiges

Lesetheater. Handbuch zu Theorie und Praxis. Opladen; Berlin; Toronto : Verlag Barbara Budrich 2017, S. 7-14 - URN: urn:nbn:de:0111-pedocs-209837 - DOI: 10.25656/01:20983

https://nbn-resolving.org/urn:nbn:de:0111-pedocs-209837

https://doi.org/10.25656/01:20983

in Kooperation mit / in cooperation with:

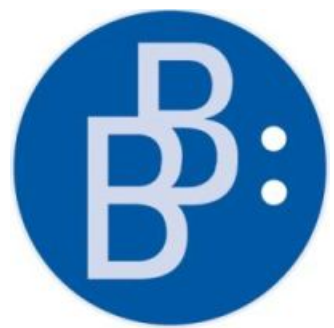

https://www.budrich.de

\section{Nutzungsbedingungen}

Gewährt wird ein nicht exklusives, nicht übertragbares, persönliches und beschränktes Recht auf Nutzung dieses Dokuments. Dieses Dokument ist ausschließlich für den persönlichen, nicht-kommerziellen Gebrauch bestimmt. Die Nutzung stellt keine Übertragung des Eigentumsrechts an diesem Dokument dar und gilt vorbehaltlich der folgenden Einschränkungen: Auf sämtlichen Kopien dieses Dokuments müssen alle Urheberrechtshinweise und sonstigen Hinweise auf gesetzlichen Schutz beibehalten werden. Sie dürfen dieses Dokument nicht in irgendeiner Weise abändern, noch dürfen Sie dieses Dokument für öffentliche oder kommerzielle Zwecke vervielfältigen, öffentlich ausstellen, aufführen, vertreiben oder anderweitig nutzen.

Mit der Verwendung dieses Dokuments erkennen Sie die Nutzungsbedingungen an.

\section{Kontakt / Contact:}

peDOCS

DIPF | Leibniz-Institut für Bildungsforschung und Bildungsinformation Informationszentrum (IZ) Bildung

E-Mail: pedocs@dipf.de

Internet: www.pedocs.de

\section{Terms of use}

We grant a non-exclusive, non-transferable, individual and limited right to using this document.

This document is solely intended for your personal, non-commercial use. Use of this document does not include any transfer of property rights and it is conditional to the following limitations: All of the copies of this documents must retain all copyright information and other information regarding legal protection. You are not allowed to alter this document in any way, to copy it for public or commercial purposes, to exhibit the document in public, to perform, distribute or otherwise use the document in public.

By using this particular document, you accept the above-stated conditions of use. 
Sabine Kutzelmann Ute Massler Klaus Peter Kristina Götz Angelika Ilg (Hrsg.)

\section{Mehrsprachiges Lesetheater}

Handbuch zu Theorie und Praxis 
Mehrsprachiges Lesetheater 

Sabine Kutzelmann • Ute Massler • Klaus Peter • Kristina Götz • Angelika Ilg (Hrsg.)

\section{Mehrsprachiges Lesetheater}

Handbuch zu Theorie und Praxis

Verlag Barbara Budrich

Opladen • Berlin • Toronto 2017 
Bibliografische Information der Deutschen Nationalbibliothek

Die Deutsche Nationalbibliothek verzeichnet diese Publikation in der Deutschen Nationalbibliografie; detaillierte bibliografische Daten sind im Internet über http://dnb.d-nb.de abrufbar.

Gefördert durch

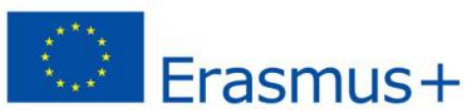

Schulbildung

Dieses Projekt wurde mit Unterstützung der Europäischen Kommission finanziert. Die

Verantwortung für den Inhalt dieser Veröffentlichung (Mitteilung) trägt allein der Verfasser; die Kommission haftet nicht für die weitere Verwendung der darin enthaltenen Angaben.

This project has been funded with support from the European Commission. This publication reflects the views only of the author, and the Commission cannot be held responsible for any use which may be made of the information contained therein.

Gedruckt auf säurefreiem und alterungsbeständigem Papier.

Alle Rechte vorbehalten.

C 2017 Verlag Barbara Budrich, Opladen, Berlin \& Toronto

www.budrich-verlag.de

$$
\text { ISBN 978-3-8474-2021-4 }
$$

eISBN 978-3-8474-1109-3

Das Werk einschließlich aller seiner Teile ist urheberrechtlich geschützt. Jede Verwertung außerhalb der engen Grenzen des Urheberrechtsgesetzes ist ohne Zustimmung des Verlages unzulässig und strafbar. Das gilt insbesondere für Vervielfältigungen, Übersetzungen, Mikroverfilmungen und die Einspeicherung und Verarbeitung in elektronischen Systemen.

Umschlaggestaltung: Bettina Lehfeldt, Kleinmachnow - www.lehfeldtgraphic.de

Lektorat und Satz: Ulrike Weingärtner, Gründau

Titelbildnachweis: Stillaufnahme aus dem MELT Film „LOUD, DEUTLICH, LENTEMENT: Mit

Mehrsprachigem Lesetheater Sprachgrenzen überwinden“ (s. Projektwebsite: http://melt-multilingualreaders-theatre.eu/ )

Druck: paper \& tinta, Warschau

Printed in Europe 


\section{Inhalt}

Das Projekt Mehrsprachiges Lesetheater: Ausgangslage, Zielsetzung

und praxisrelevante Ergebnisse der Entwicklung .................

Sabine Kutzelmann, Ute Massler und Robert Hendel

\section{Teil A}

\section{Forschungsmethodik}

1 Design-Based Research: Eine Forschungsstrategie zur Entwicklung nachhaltiger Innovationen in der schulischen Praxis . . . . . . . . . . . . Sabine Kutzelmann und Kristina Götz

2 Die schulischen Erprobungskontexte des MELT-Projekts . . . . . . . . . . . 32 Kristina Götz, Robert Hendel, Sabine Kutzelmann und Klaus Peter

3 Mehrsprachiges Lesetheater: Ergebnisse zur Akzeptanz dieser

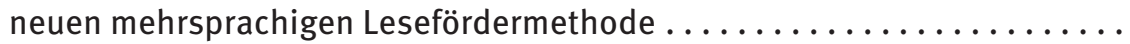
Kristina Götz, Sabine Kutzelmann und Ute Massler

Teil B

Theoretische Grundlagen des

Entwicklungs- und Forschungsprozesses

1 Leseflüssigkeit und Lesemotivation: Die beiden Förderdimensionen des Mehrsprachigen Lesetheaters . . . . . . . . . . . . . . . Kristina Götz und Sabine Kutzelmann

2 Das Unterrichtsdesign Mehrsprachiges Lesetheater aus linguistischer Sicht.

Klaus Peter und Dominik Unterthiner

3 Vom Readers' Theatre über interlinguale Literatur zum Mehrsprachigen Lesetheater: Die didaktisch-methodischen Leitlinien der Gestaltung im Kontext der Mehrsprachigkeitsdidaktik ................. Ute Massler und Sabine Kutzelmann

\section{Teil C}

\section{Das didaktisch-methodische Design:}

\section{Theoretische Grundlagen und Anleitung für die Praxis}

1 Mehrsprachige Lesetheaterstücke: Konzeption der Lehr-Lern-Materialien . . . Angelika Ilg, Franz Ludescher und Seraina Paul

2 Mehrsprachiges Vorlesen durch die Lehrperson als Design-Komponente des Mehrsprachigen Lesetheaters . . . . . . . . . . . . . . . . . .

Ute Massler und Kerstin Theinert 
3 Miteinander sind alle Stars - Die Bedeutung des kooperativen Lernens

im Mehrsprachigen Lesetheater.

120

Sabine Kutzelmann und Kerstin Theinert

4 Literarisches Lernen als Design-Komponente des

Mehrsprachigen Lesetheaters

Angelika Ilg und Kerstin Theinert

5 Die zentralen Lehr-Lern-Prozesse des Mehrsprachigen Lesetheaters:

Eine Anleitung für die Praxis

Sabine Kutzelmann, Ute Massler, Klaus Peter unter Mitarbeit von

Anne-Marie Antony, Stefanie Arnold, Kurt Benedikt, Andrea Büchel,

Stefanie Blodnig, Tessy Gollère, Robert Hendel und Christian Mertens

\section{Teil D}

\section{Curriculum Lehrerfortbildung}

1 Professionswissen und Einstellungen von Sprachlehrpersonen zur

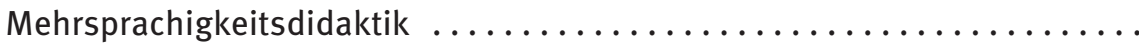
Klaus Peter und Dominik Unterthiner

2 Sprach- und Fachgrenzen überwinden: Die didaktische und strategische Konzeption des Fortbildungscurriculums zur Lesefördermethode

Mehrsprachiges Lesetheater ......................... 168

Sabine Kutzelmann und Ute Massler

Anhang 1) Anleitung zur Dramatisierung von mehrsprachigen Lesetheatern am Beispiel von Bend it like Beckham . . . . . . . . . . . . . . . . . 181 Anhang 2) Lesetheater Heidi . . . . . . . . . . . . . . . . . . . . . . 185

Anhang 3) Lesetheater Luina - Die Seejungfrau vom Bodensee . . . . . . . . . . . 189

Anhang 4) Lesetheater Nasreddin Hodscha . . . . . . . . . . . . . . . . . . . . 191

Anhang 5) Lesetheater Dr Jekyll and Mr Hyde . . . . . . . . . . . . . . . . . 195

Anhang 6) Toolbox: Dramapädagogik ...................... 196

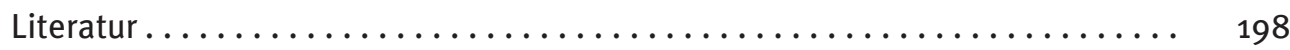

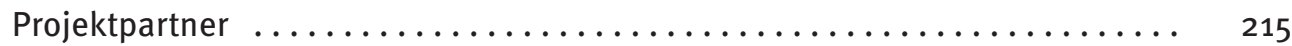

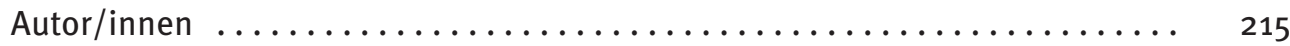




\title{
Das Projekt Mehrsprachiges Lesetheater: Ausgangslage, Zielsetzung und praxisrelevante Ergebnisse der Entwicklung
}

\author{
Sabine Kutzelmann, Ute Massler und Robert Hendel
}

\section{$1 \quad$ Ausgangslage und Zielsetzung}

Das Entwicklungs- und Forschungsprojekt Mehrsprachiges Lesetheater zur Förderung der Leseflüssigkeit und Lesemotivation (MELT), das von der Europäischen Kommission durch das Förderprogramm Erasmus+ finanziert wurde, entwickelte eine mehrsprachige Lesefördermethode, die integrativ die schulisch relevanten Sprachen einbezieht ${ }^{1}$. Mehrsprachiges Lesetheater baut auf dem Readers' Theatre auf, einer Lautlesemethode, die bislang kaum in der Schulpraxis der deutschsprachigen Länder Europas eingesetzt wird. Um Lesetheater in der Schule umzusetzen, werden literarische Texte in dialogische Vorlesetexte umgewandelt. Das gestaltende Vorlesen dieser Texte wird in kooperierenden Schülerarbeitsgruppen trainiert und abschließend aufgeführt. Für die Konzeption des Mehrsprachigen Lesetheaters wurde die traditionellerweise einsprachige, auf die Schulsprache fokussierte Methode zugunsten eines zwei- bzw. mehrsprachigen, sprachübergreifenden Ansatzes weiterentwickelt, der neben den Schul- und Fremdsprachen auch die Integration von Migrationssprachen vorsieht. Des Weiteren wurde die Methode um die Komponenten mehrsprachiges Vorlesen der Lehrperson und literarisches Lernen ergänzt. MELT lagen folgende Annahmen zugrunde: (1) Aktuelle Systematiken der Lesedidaktik (z. B. das „Mehrebenenmodell des Lesens“ von Rosebrock/Nix 2014) verweisen auf verschiedene Ebenen, die relevante Anknüpfungspunkte für schulische Lesefördermethoden bieten. (2) Aufgrund zunehmend international agierender Firmen, erhöhter Mobilität und von der Globalisierung betroffener Arbeitsplätze thematisieren EU-/Bildungspolitiker/innen und Forschende die gezielte Förderung der Fremdsprachenkompetenz und der individuellen Mehrsprachigkeit bei Lernenden (European Commission: 2012, 33 und 35). (3) Gleichzeitig fordern die Curricula in Österreich, Deutschland, Luxemburg und der Schweiz, Synergien in den Lehrund Lernprozessen des Schul- und Fremdsprachunterrichts zu schaffen.

Im MELT-Projekt wurden quantitative und qualitative Methoden zur Gewinnung und Auswertung von Daten kombiniert eingesetzt, um verschiedene Zusammenhänge zu analysieren: (1) die Akzeptanz der Methode in der schulischen Praxis, (2) die Entwicklung der Leseflüssigkeit in Schul- und Fremdsprachen aufgrund der Arbeit mit dem Mehrsprachigen Lesetheater sowie (3) der Einfluss von MELT auf die Lesemotivation. Die Entwicklung und Erprobung der Methode erfolgte nach dem Design-BasedResearch-Ansatz (DBR). Wie die englische Bezeichnung design-based es nahelegt, ging

1 In diesem Handbuch wird aus Gründen der besseren Lesbarkeit lediglich der Kurztitel des Entwicklungs- und Forschungsprojekts verwendet. Der ausführliche Titel lautet: Mehrsprachiges Lesetheater zur Förderung von Lesemotivation und Leseflüssigkeit (MELT). 
es im Projekt um die Gestaltung der Lesefördermaßnahme Mehrsprachiges Lesetheater ${ }^{2}$. Spezifisch am DBR-Ansatz ist, dass für die Ausarbeitung einer Maßnahme mehrere sich wiederholende Phasen durchlaufen werden: Design, praktische Erprobung und Evaluation, Analyse und Redesign der Maßnahme. Mithilfe dieser iterativen Vorgehensweise wurde MELT mit Lehrkräften und Schulklassen der Primar- und der Sekundarstufe I in den vier beteiligten Partnerländern - Deutschland, Luxemburg, Österreich und der Schweiz - sukzessive optimiert und auf ihre Akzeptanz hin untersucht.

Das Projekt strebte an, sowohl einen Beitrag zur theoretischen Fundierung eines mehrsprachigen Ansatzes zu leisten, als auch ein praktikables Produkt für einen mehrsprachig ausgerichteten Unterricht vorzuweisen. Dies bedeutete konkret:

- in Kooperation mit Lehrkräften ein didaktisch-methodisches Design zu entwickeln, das einerseits die Leseflüssigkeit und -motivation und damit die Lesekompetenz der Lernenden fördert und andererseits die Akzeptanz bei Lehrkräften sowie Lernenden erhöht, sich auf Lernprozesse in mehrsprachigen Unterrichtssettings einzulassen,

- in diesem Zusammenhang auf andere Kontexte übertragbare Prototypen von mehrsprachigen Lesetheaterstücken im Sinne von Good-practice-Beispielen zu entwickeln,

- begleitende Unterrichtsmaterialien für den Deutsch-, Englisch- und den Französischunterricht zu entwickeln, die eine Differenzierung nach Leistungsniveaus und den Einbezug von Migrationssprachen ermöglichen, zu erproben und zu optimieren,

- in enger Zusammenarbeit mit Unterrichtspraktiker/innen ein Fortbildungscurriculum auszuarbeiten, um Lehrkräften für die Umsetzung des Mehrsprachigen Lesetheaters die benötigten Handlungskompetenzen zu vermitteln,

- ein qualitatives Forschungsinstrumentarium zu entwickeln, das verschiedene Arten von Daten erhebt (z. B. Fragebogen, teilnehmende Beobachtung), die möglichst umfassend über die Akzeptanz des Mehrsprachigen Lesetheaters aus Sicht der Lehrkräfte und ihrer Klassen Auskunft geben,

- ein qualitatives und quantitatives Forschungsinstrumentarium zu entwickeln, um bei den Lernenden die Lesemotivation und die Leseflüssigkeit (insbesondere des prosodischen, d. h. des gestaltenden Lesens) zu erheben und um Synergieeffekte zwischen den Sprachen sichtbar zu machen und damit zur Theorieentwicklung eines mehrsprachigen Unterrichts beizutragen, und

- damit einen Beitrag zur theoretischen Fundierung einer Didaktik der Mehrsprachigkeit zu liefern, indem sich im Sinne von DBR das MELT-Design solide auf Erkenntnisse der schul- und fremdsprachlichen Leseforschung sowie der Sprachlehr- und -lernforschung abstützt.

Das Projekt Mehrsprachiges Lesetheater richtet sich an folgende Zielgruppen:

2 Um das forschungsmethodische Vorgehen auch begrifflich zu akzentuieren, wird neben dem Begriff der Lesefördermaßnahme im Handbuch auch vom didaktisch-methodischen Design gesprochen. 
- Lernende der 5. bis 8. Klassenstufe mit Deutsch als Schulsprache, insbesondere auch schwächere Lernende (beim Anspruchsniveau der verschiedenen Leserollen wird differenziert), und Lernende mit Deutsch als Zweitsprache: Kritisches Alter bezüglich Leseleistung und Fremdsprachenlernmotivation;

- Lehrkräfte, die Deutsch und mindestens eine Fremdsprache in der 5. bis 8. Klasse unterrichten, da eine konsequente Verortung der Mehrsprachigkeitsdidaktik auf diesen Stufen noch nicht im Klassenzimmer angekommen ist. Schul- und Fremdsprache werden nach wie vor mehrheitlich isoliert unterrichtet;

- Lehrerfortbildner/innen: Auch in der Lehrerfortbildung sind mehrsprachige Ansätze wenig verbreitet;

- Curriculumsverantwortliche (Schulverwaltungen, Lehrplan- und Lehrmittelautor/innen): In Lehrplänen ist die mehrsprachige Sicht bereits verankert, doch fehlen konkrete didaktische Mittel zur Umsetzung;

- Hochschullehrkräfte: Bislang werden an den Lehrerausbildungsinstitutionen die Lehr- und Lernveranstaltungen der Fachdidaktiken (in Schul- und Fremdsprachen) isoliert unterrichtet, d. h. nicht in Bezug auf Mehrsprachigkeit bzw. mehrsprachiges Lernen koordiniert. Mit dem EU-Projekt wird ein Beitrag zur Theoriebildung einer profilierten Didaktik der Mehrsprachigkeit geleistet.

\section{Praxisrelevante Ergebnisse der Entwicklung}

Um die nachhaltige Nutzung des Mehrsprachigen Lesetheaters zu sichern, wurden verschiedene praxisrelevante Maßnahmen konzipiert und umgesetzt. Hierzu zählen neben der Publikation des vorliegenden Handbuchs, ein didaktisch-methodischer Kommentar für Lehrpersonen (vgl. Teil C, Kap. 5), ein Lehrfilm, ein Lehrerfortbildungscurriculum (vgl. Teil D, Kap. 2) sowie ein Webportal, das die erarbeiteten Lehr- und Lernmaterialien enthält (siehe unten). Alle genannten Maßnahmen verdeutlichen den mehrteiligen und komplexen Lehr-Lern-Prozess des Mehrsprachigen Lesetheaters und erleichtern seine Umsetzung. Durch die konstante Verfügbarkeit dieser praktischen Ergebnisse auf dem Webportal tragen sie zur Verbreitung der Lesefördermethode bei.

Lehr- und Lernmaterialien: Im Projekt wurden rund 30 mehrsprachige Lesetheaterstücke für verschiedene Sprachenkombinationen und Klassenstufen erstellt und erprobt, die sowohl auf aktueller Kinder- und Jugendliteratur als auch Klassikern basieren. Aus Gründen des Urheberrechtsschutzes steht Lehrpersonen bislang nur eine Auswahl dieser Lehr- und Lernmaterialien auf dem Webportal zur Verfügung. Eine größere Bereitschaft von Verlagen, Ausschnitte aus interessanten literarischen Vorlagen aus didaktischen Gründen zu mehrsprachigen Lesetheaterstücken zur Adaption und zur Weitergabe über das Webportal zur Verfügung zu stellen, wäre wünschenswert gewesen. Bedauerlicherweise waren hierfür zumeist nur kleinere Verlage oder einzelne Autor/innen zu gewinnen.

Didaktisch-methodischer Kommentar: Der Kommentar beschreibt die acht zentralen Schritte des Mehrsprachigen Lesetheaters und begründet die jeweilige Funktion didaktisch (vgl. Teil C, Kap. 5). Außerdem werden weiterführende methodische Anregungen im Sinne von good practice gegeben, die auf den praktischen Erfahrungen der Kooperationslehrpersonen, die sie in den Erprobungen sammeln konnten, basieren; sie sollen 
Lehrpersonen mit ihren Klassen eine methodisch vielfältige Durchführung von MELT ermöglichen.

Lehrfilm: Im Auftrag des luxemburgischen Bildungsministeriums (MENJE/SCRIPT) erarbeitete das DPAV (Documentation et production audio-visuelle), einen Dokumentarfilm zum Mehrsprachigen Lesetheater. Zu sehen sind die Schüler/innen und Lehrpersonen aus den vier Partnerschulen. Der Film dokumentiert und kommentiert die acht Schritte des Lehr- und Lernprozesses und gibt dabei auch die Sicht der Schüler/innen und der Kooperationslehrkräfte hierauf wieder. Er kann sowohl in der Weiterbildung von Lehrpersonen als auch in der Ausbildung von Studierenden an Pädagogischen Hochschulen und Universitäten eingesetzt werden. Der Film wurde beim MELT-Multiplier-Event im Lënster Lycée in Luxemburg am 26.11.2016 öffentlich vorgestellt und kann unter folgender Adresse aufgerufen werden: http://melt-multilingual-readers-theatre.eu/.

Webportal: Das Webportal informiert über alle für das Projekt relevanten Inhalte, Ziele und Aktivitäten. Kostenlos zur Verfügung stehen dort eine Auswahl der entwickelten Lehr- und Lernmaterialien, der didaktisch-methodische Kommentar sowie der Lehrfilm. Neben der deutschen Fassung liegt das Webportal auch in der englischen Version vor: http://melt-multilingual-readers-theatre.eu/material1/.

Lehrerfortbildungscurriculum: Um die Einführung von MELT in den verschiedenen Bildungsregionen zu unterstützen, wurde auch ein Fortbildungscurriculum konzipiert; es gibt Fortbildungsverantwortlichen didaktische Leitideen an die Hand, damit sie Lehrpersonen erfolgreich zum Mehrsprachigen Lesetheater weiterbilden können. Die Grundlagen des Curriculums bilden einerseits inhaltlich die Ergebnisse des vorliegenden Projekts und andererseits didaktisch die Ergebnisse der Lehr- und Lernforschung zu den Merkmalen wirksamer Weiterbildung (vgl. Teil D, Kap. 2).

Multiplierevents: Während der Projektlaufzeit wurden eine internationale Lehrerfortbildung im Raum Luxemburg-Frankreich (26.11.2016, Lënster Lycée, Junglinster, Luxemburg) sowie eine im Raum Deutschland-Österreich-Schweiz durchgeführt (19.5.2017, Pädagogische Hochschule Weingarten, Deutschland). Die dritte Multiplikatorenveranstaltung stellte eine internationale wissenschaftliche Fachtagung dar (20.5.2017, Pädagogische Hochschule Weingarten, Deutschland). Hierbei wurden zum einen neueste Ergebnisse der Grundlagenforschung zur Entwicklung und Förderung der Lesefertigkeit in unterschiedlichen Kontexten und mit unterschiedlichen Lernenden präsentiert. Zum anderen wurden praxisbasierte Studien vorgestellt, die didaktisch-methodische Konzepte für eine mehrsprachige Leseförderung in der Primarstufe und der Sekundarstufe I erarbeiten. Die Beiträge werden im Narr Verlag in der Reihe Studienbücher publiziert (Kutzelmann/Massler in Druck).

\section{Aufbau des Handbuches}

Teil A des Handbuchs geht auf die Forschungsmethodik, die schulischen Erprobungskontexte sowie die zentralen Ergebnisse zur Akzeptanz des didaktisch-methodischen Designs ein.

Kapitel A1 stellt in einem ersten Teil die Zielsetzungen und Merkmale des Design-Based-Research-Ansatzes vor, die für das Vorgehen im Projekt maßgeblich waren. 
Außerdem wird auf die Phasen des DBR-Entwicklungs- und Forschungsprozesses sowie den spezifischen Beitrag der am Projekt beteiligten Praktiker/innen eingegangen. In einem zweiten Teil werden zentrale Themen der Akzeptanzforschung vorgestellt und das Akzeptanzmodell von Venkatesh et al. (2003) eingeführt, das als theoretische Grundlage für die Akzeptanzüberprüfung diente. Anschließend wird konkretisiert, wie das Kriterium der Akzeptanz sowohl für die formative als auch für die summative Evaluation des Entwicklungs- und Forschungsprozesses genutzt wurde.

Kapitel A2 beschreibt die Kontexte, unter denen das Mehrsprachige Lesetheater in den Partnerländern Deutschland, Luxemburg, Österreich und Schweiz erprobt wurde. Dabei wird auf die unterschiedlichen Schulformen, Altersgruppen und Rahmenbedingungen eingegangen. Auch werden die Kooperationsklassen mit Blick auf ihre Lesefertigkeiten (Leseflüssigkeit) und Lesemotivation zu Beginn der Hauptstudie beschrieben.

In A3 werden die Ergebnisse zur Akzeptanz des Unterrichtsdesigns als mehrsprachige Lesefördermethode aus der Perspektive der Lehrpersonen dargestellt. Dabei wird vorwiegend auf ihre Beurteilung der Nützlichkeit des Designs, der Lehr-Lern-Materialien und einzelner Design-Komponenten eingegangen. Des Weiteren werden der Aufwand, den die Anwendung des Unterrichtdesigns bedeutet, sowie der Verwendung zuträgliche Rahmenbedingungen erörtert, bevor die Nutzungsintentionen der Lehrpersonen besprochen werden. Im abschließenden Fazit werden die Ergebnisse in Bezug zu den Erprobungskontexten diskutiert und ein Ausblick zum Mehrsprachigen Lesetheater in der Praxis gegeben.

Teil B erörtert die theoretischen Grundlagen des Entwicklungs- und Forschungsprozesses, wobei B1 zunächst auf die Förderung von Leseflüssigkeit und Lesemotivation im MELT-Konzept fokussiert. Hierfür wird eingangs eine Zusammenfassung des Forschungsstands zur Bedeutung der beiden Förderdimensionen in der Erst- als auch in der Fremdsprache gegeben. Darauf basierend wird dargestellt, wie das Design konzipiert wurde, um nicht nur positiv auf die Leseflüssigkeit zu wirken, sondern auch motivierend das Leseverhalten der Schüler/innen zu beeinflussen.

Die linguistischen Grundlagen des Unterrichtsdesigns thematisiert Kapitel B2. Nach einem kurzen Überblick über unterschiedliche Definitionen und Modelle von Mehrsprachigkeit im linguistischen Diskurs beleuchtet das Kapitel die Rolle der Schulsprache sowie weiterer Sprachen im Fremdsprachenunterricht. Darauf aufbauend wird ein Überblick über die in der Linguistik verorteten und auch für das Mehrsprachige Lesetheater relevanten mehrsprachigkeitsdidaktischen Ansätze wie Interkomprehension oder Translanguaging gegeben. Abschließend werden vor diesem Hintergrund Chancen und Grenzen von MELT aus linguistischer Sicht diskutiert.

Die Fördermethode ist Inhalt von Kapitel B3. Hierfür wird zunächst auf die beiden theoretisch-konzeptuellen Leitlinien fokussiert, die bei der Gestaltung des MELTDesigns als Ganzes aus mehrsprachigkeitsdidaktischer Perspektive in den verschiedenen Phasen des DBR-Entwicklungs- und Forschungsprozesses berücksichtigt wurden: (1) Readers' Theatre als Grundlage des didaktisch-methodischen Designs und (2) die mehrsprachigen Lernmaterialien. Abschließend verortet das Kapitel das Mehrsprachige Lesetheater im Kontext der Mehrsprachigkeitsdidaktik.

Teil C beschreibt die theoretischen Grundlagen, die für die Konzeption der Designelemente leitend waren sowie die daraus resultierende Ausgestaltung der ver- 
schiedenen Elemente. Des Weiteren wird ausgeführt wie das MELT-Design und seine Komponenten in der Praxis eingesetzt werden.

Kapitel C1 befasst sich mit der Konzeption der Lehr- und Lernmaterialien im Projekt Mehrsprachiges Lesetheater. Nach der Vorstellung der Beweggründe für die verwendeten Sprachenkombinationen werden die Prinzipien erläutert, auf deren Basis die Lehr-Lern-Materialien entwickelt wurden. Gleichzeitig wird die Vorgangsweise beim Prozess des Dramatisierens beschrieben. Aufgrund des mehrsprachigen Charakters des Designs wird der Konstruktion der fremdsprachlichen Textteile besondere Aufmerksamkeit geschenkt. In diesem Zusammenhang wird auch ein Modell zur Integration weiterer Herkunftssprachen in bestehende Lesetheater präsentiert. Der letzte Abschnitt des Kapitels widmet sich den Audiodateien, die zu den mehrsprachigen Lesetheaterskripts erstellt wurden und erklärt deren Funktionen und Einsatzmöglichkeiten im Unterricht.

Kapitel C2 erläutert, welche Bedeutungen und Funktionen dem Vorlesen durch die Lehrperson im MELT-Unterrichtsdesign zukommen und wie es die Wahrnehmung der Schüler/innen verändert, wenn ihnen die Lehrperson mehrsprachig vorliest. Nach der Beschreibung der Funktionen des Vorlesens wird auf die Auswahl und Gestaltung der Vorlesetexte eingegangen. Anschließend werden die theoretischen Grundlagen für die Konzeption des Vorlesens dargestellt und dabei der spezifische Mehrwert des Vorlesens in Fremd- und Schulsprache herausgearbeitet. Besondere Aspekte des gestaltenden Vorlesens beschließen das Kapitel.

Die konzeptuell-theoretischen Grundlagen des kooperativen Lernens sind Thema von Kapitel C3. Hierfür werden zuerst einige wesentliche Merkmale erläutert, die gerade auch in Hinblick auf die Realisierung des kooperativen Lernens in der Unterrichtspraxis relevant sind. Anschließend wird auf die besondere Rolle der Lehrperson beim kooperativen Lernen, vor allem bei der Einführung in ein mehrsprachiges Lesetheaterstück, eingegangen. Da anzunehmen ist, dass durch kooperatives Lernen auch die im MELT-Design angestrebten sozialen und kommunikativen Kompetenzen gefördert werden, schließt das Kapitel mit einigen zentralen Ergebnissen zur Wirksamkeit kooperativen Lernens.

Die im Unterrichtsdesign integrierten Formen literarischen Lernens in Gestalt von Dramapädagogik und Anschlusskommunikation werden in Kapitel C4 präsentiert. Nach einer Begriffsbestimmung und einer Einführung zum Thema werden die theoretischen Grundlagen der Bereiche Dramapädagogik und Anschlusskommunikation sowie deren Funktionen und Relevanz erläutert. Im Anschluss daran werden jeweils im Kontext des Mehrsprachigen Lesetheaters erprobte Übungen beschrieben. Dabei werden Umsetzungsmöglichkeiten unter Einbeziehung der schulisch geförderten Fremdsprachen erläutert. Der Beitrag schließt mit Gestaltungsprinzipien, die aus den Unterrichtserprobungen für das literarische Lernen im MELT-Design resultieren.

Im Laufe des Entwicklungs- und Forschungsprojekts kristallisierten sich aufgrund der formativen Evaluation der Erprobungszyklen acht Schritte heraus, die den Ablauf des Lehr-Lern-Prozesses im MELT-Design didaktisch und methodisch sinnvoll strukturieren. Kapitel C5 beschreibt diese zentralen Schritte und begründet ihre jeweilige Funktion didaktisch. Außerdem gibt es weiterführende methodische Anregungen im Sinne von good practice. 
Teil D beschreibt abschließend das Professionswissen von Lehrpersonen im Bereich der Mehrsprachigkeitsdidaktik und präsentiert das Fortbildungscurriculum zum Mehrsprachigen Lesetheater.

Gegenstand von Kapitel D1 sind das Professionswissen sowie die Einstellungen von Lehrpersonen gegenüber mehrsprachigkeitsdidaktischen Konzepten und Verfahren. Nach einleitenden Erläuterungen zum Zusammenhang von Professionswissen bzw. Einstellungen und konkretem Lehrerhandeln gibt das Kapitel einen Überblick über aktuelle empirische Untersuchungen zu Haltungen und Überzeugungen von Lehrpersonen in Bezug auf mehrsprachigkeitsdidaktische Unterrichtskonzepte. Auf dieser Grundlage werden die daraus resultierenden Konsequenzen für die Lehreraus- und -fortbildung im Allgemeinen sowie für das Lehrerfortbildungscurriculum Mehrsprachiges Lesetheater im Speziellen diskutiert. Kapitel D2 beschreibt die inhaltliche, didaktische und strategische Konzeption des Fortbildungscurriculums zum Mehrsprachigen Lesetheater. Auf der Basis des Angebots-Nutzungsmodell von Lipowsky (2014: 515) wird erläutert, welche strukturellen und didaktischen Merkmale im Allgemeinen und mit Hinblick auf das Fortbildungscurriculum dazu beitragen, dass Fortbildungsangebote wirksam werden. In einem zweiten Teil werden die verschiedenen Phasen vorgestellt, die für die Planung und Gestaltung von Fortbildungsmaßnahmen zum Mehrsprachigen Lesetheater leitend sind.

\section{4 \\ Danksagung}

Das Forschungsprojekt wurde 2014 bis 2017 von der Europäischen Kommission als Erasmus+-Projekt gefördert. Der EU gilt unser besonderer Dank. Das Regierungspräsidium Tübingen förderte das Projekt durch die Genehmigung von Erlassstunden für die deutsche Kooperationslehrkraft, auch hierfür sind wir sehr dankbar. Ebenso danken wir den beteiligten Hochschulen und dem SCRIPT für die großzügige finanzielle und organisatorische Unterstützung der Projekttätigkeiten.

Während des Projekts erprobten die kooperierenden Lehrkräfte und ihre Schüler/innen das MELT-Konzept mit sehr großem Engagement in ihrer Unterrichtspraxis. Ihr Beitrag trug wesentlich zum Gelingen des Entwicklungs- und Forschungsprozesses bei und ist von größter Bedeutung. Daher danken wir folgenden Kooperationsschulen und Lehrkräften sehr herzlich: Vorarlberger Mittelschule Bregenz Vorkloster (Österreich): Kurt Benedikt, Stefanie Blodnig; Lënster Lycée (Luxemburg): Tessy Gollère, Christian Mertens; Pestalozzischule Rorschach (Schweiz): Andrea Büchel; Realschule Weingarten (Deutschland): Stefanie Arnold.

Im Projektteam arbeitete neben den Autor/innen dieses Bandes und den genannten Lehrpersonen auch Anne-Marie Antony. Auch ihr danken wir sehr herzlich für ihr unermüdliches und kompetentes Engagement.

Wir danken auch Prof. Dr. Michael Link, der uns als profunder Kenner des DBR-Ansatzes bei der forschungsmethodischen Konzeption des MELT-Projekts beriet.

Den Mitarbeitenden des SCRIPT DPAV danken wir für ihren unermüdlichen und großartigen Einsatz bei der Erstellung des Lehrfilmes: Anne Schiltz/Tom Alesch (Regie); Pol Linden (Bild), Marc Mergen (Ton und Musik), Anouk Wagener (Off-Stimme), Marc Recchia (Schnitt), Carlo Thiel (Farbkorrektur), Tom Schartz (Grafik). Unser Dank 
gilt auch Annette Démarais Stickel; sie leitete die theaterpädagogischen Workshops an den beiden Multiplierevents.

Im Rahmen des Projekts arbeiteten zahlreiche Studierende der Partnerhochschulen als wissenschaftliche Hilfskräfte bzw. studentische Projektmitarbeitende mit. Auch ihnen danken wir herzlich dafür, dass sie ihre Perspektive und ihr Verständnis einbrachten und mit uns teilten. Insbesondere möchten wir Jon Duri Cajochen, Selina Huser, Tamara Mai, Majken Marten und Mathias Perzl nennen.

Wir danken den folgenden Verlagen und Autor/innen, die uns, zum Teil zeitlich begrenzt, das Adaptieren von Auszügen ihrer Werke wie auch die kostenlose Veröffentlichung der mehrsprachigen Lesetheaterstücke gestatteten: Auer, Martin: Balaban, Balaban-Smashwords, Ich das machen; Burgess, Melvin (Pearson-Verlag): Billy Eliott; Kleiner, Sabine/Steinmayer, Stefanie (Verlag Schwäbisches Meer): Luina - Die Seejungfrau vom Bodensee; Luina - Die Seejungfrau erobert den Bodensee; Knauss, Susanne: Tatort Schule; Knauss, Susanne/Selle, Martin (Amrun Verlag): Dark Night - draußen allein!; Time Twister: Die unglaubliche Reise ins Ich; Time Twister: Madagaskar - Insel der Rätseltiere; Die Wahrheit über Derek Foster; Herr Bogomil und Mister Pumbuli - Das Geheimnis der Mumie; Niebisch, Jackie (DTV): Die Kleinen Wilden. Schuker, Klaus (Allitera Verlag): Paul, Tabea und das blaue Messer; Paul, Tabea und das Friedhofsgespenst; Paul, Tabea und die brennende Hütte. Des Weiteren danken wir Studierenden unserer Hochschulen, die im Rahmen von Studienarbeiten Lesetheatertexte erstellt oder adaptiert haben: Debora Adolphs: Das Dschungelbuch; Katja Broger: Die Wilden Hühner auf Klassenfahrt; Annette Tobler: Ronja Räubertochter. Hümeyra Kongur: Rico, Oskar und die Tieferschatten. Annika Hartkorn, Janina Schwarz: Tatort Schule; Tanja Willmann: Die Fischapp im Smartphone; Madeleine Rutschmann: Der Froschkönig und der eiserne Heinrich; Jennifer Denkinger: Hänsel und Gretel REMASTERED; Sarah Herter, Kujtesa Krasniqi, Selina Brugger, Lena Traub: Little Red Riding Hood; Annika Hartkorn: Luina - Die Seejungfrau vom Bodensee; Miriam Adt: The Tales of Nasreddin Hodscha; Selina Wagner, Carina Kromer, Miriam Adt, Katharina Abel, Kristina Karij, Fiona Emme, Tamara Mai, Robin Raff: The Canterville Ghost; Anja Untersander, Samira Tüxsen: Das Friedhofsgespenst.

St. Gallen, Weingarten, Luxemburg im April 2017 